\title{
ELABORACIÓN Y VALIDACIÓN DE UN INSTRUMENTO DIAGNÓSTICO PARA LA PERCEPCIÓN DE LAS COMPETENCIAS EMOCIONALES EN ESTUDIANTES UNIVERSITARIOS DE EDUCACIÓN
}

\author{
DEVELOPMENT AND VALIDATION OF A DIAGNOSTIC INSTRUMENT \\ FOR EMOTIONAL PERCEPTION OF COMPETENCE IN COLLEGE \\ STUDENTS OF EDUCATION \\ María Josefa Iglesias Cortizas* \\ Universidad de A Coruña
}

\section{RESUMEN}

Existe un creciente interés en el diseño de instrumentos que posibiliten la identificación y evaluación de las competencias profesionales, personales o sistémicas, diseñadas en el Proyecto Tuning, bajo las directrices de la Declaración de Bolonia (1999), que regulan el Espacio Europeo de Educación Superior. El artículo tiene como propósito aportar un mayor conocimiento sobre las competencias emocionales en jóvenes universitarios de educación mediante la identificación de factores que permitan proponer un modelo de competencias aplicable, fiable y válido para su aplicación.

En el estudio participaron 571 estudiantes universitarios con edades comprendidas e 17 y 39 años $(\mathrm{M}=20,44$ y $\mathrm{SD}=5,012)$, pertenecientes a las siete titulaciones de Ciencias de la Educación de la Universidad de La Coruña (Spain). Se diseñó un cuestionario, con una serie de elementos que pretenden identificar la percepción de las competencias emocionales, que posibilitó la recogida y posterior análisis de datos. El instrumento es de tipo Lickert con 4 categorías o alternativas de respuesta que van desde nada o nunca hasta mucho o siempre. Los niveles de fiabilidad interna $(\boldsymbol{\alpha}=0,765)$ y validez de constructo resultan aceptables.

Se propone un modelo en función de los resultados del análisis factorial (10 factores explican casi un $60 \%$ de la información), se crearon matrices de correlación con variables control para contrastar la

\footnotetext{
* María Josefa Iglesias Cortizas es Profesora Titular de la Facultad de CC de la Educación, Universidad de La Coruña. Líneas de investigación: Diagnóstico escolar. Diagnóstico e intervención didáctica del lenguaje escolar. Relaciones sociales y laborales: Alunos Sobredotados: Contributos para a Súa Identificaçao e Apoi. E-mail: majoicor@udc.es.
} 
fiabilidad de la respuesta obtenida, la validez convergente y discriminante y, finalmente, la capacidad de explicación factorial.

Palabras clave: competencias emocionales, cuestionario, estudiantes universitarios, modelo, análisis factorial, validez convergente y divergente.

\begin{abstract}
There is growing interest in designing instruments that will enable the identification and evaluation of professional skills, personal or systemic, designed in the Tuning Project, under the guidelines of the Bologna Declaration (1999), which regulate the European Higher Education Area.

The paper aims to provide a better understanding of emotional competencies in young university education by identifying factors that enable it to propose a model of competencies applicable, reliable and valid for your application.

The study involved 571 students and university students aged 17 and 39 years $(M=20.44$ and $\mathrm{SD}=5.012$ ), belonging to the seven degrees of Educational Sciences at the University of La Coruna (Spain). A questionnaire was designed with a series of elements that seek to identify the perception of emotional competencies, which enabled the collection and subsequent data analysis. The instrument is Lickert type with 4 categories or response alternatives ranging from no or never to much or always. The levels of internal reliability $(\alpha=.765)$ and construct validity are acceptable.

A model based on the results of factor analysis (10 factors explain about $60 \%$ of data), correlation matrices were created to control variables to test the reliability of the response, the convergent and discriminate validity, and finally, the explanatory power factor.
\end{abstract}

Keywords: emotional competence, questionnaire, students, model, factor analysis, convergent and divergent validity.

\title{
Introducción
}

Existe un creciente interés en el diseño de instrumentos que posibiliten la identificación y evaluación de las competencias profesionales, personales o sistémicas, diseñadas en el proyecto Tuning Educational Structures in Europe (González y Wagenaar, 2003) bajo las directrices de la Declaración de Bolonia (1999) que regulan el Espacio Europeo de Educación Superior. Entendiendo por competencia el conjunto de habilidades comprometidas en la obtención de logros y en la resolución de problemas del ámbito personal o profesional (Le Boterf, 2001; Levy-Leboyer, 1997). Hecho que se repite con la teoría de la inteligencia emocional o la capacidad para reconocer sentimientos propios y ajenos, y la habilidad para manejarlos (Goleman, 1996). En este constructo existen muchos instrumentos de recogida de información, tantos como modelos, que acertadamente Mayer, Salovey y Caruso (2000) aglutinan en dos líneas de investigación, que adoptaremos aquí:

1. Los relacionados con habilidades (Mayer y Salovey, 1997; Salovey y Meyer, 1990), centrándose en el análisis del proceso de "pensamiento acerca de los sentimientos". Es decir, la capacidad del individuo para acceder a sus emociones y crear una sintonización e integración entre sus experiencias. En esta línea están los instrumentos: el MEIS Multifactor Emotional Intelligence Scale y su última versión MSCEIT Mayer-Salovey-Caruso Emotional Intelligence Test (Mayer, Salovey y Caruso, 1999). El primero, está compuesto por 12 actividades divididas en 4 áreas que evalúan las habilidades expuestas en su teoría: percepción emocional, asimilación emocional, comprensión de las emociones y manejo emocional. Los cuatro 
componentes de MEIS y sus diferentes subescalas poseen una consistencia interna entre 0,81 y 0,96 , con un coeficiente general de 0,96. El segundo, es una versión depurada del anterior (Lopes et al., 2004). Existen dos versiones más cortas y diseñadas para el ámbito profesional como en investigación: el MSDEIT 1.1, y 2.0.

2. Los referidos al rasgo (Bar-On, 1997; Goleman, 1996, 1999) consideran la inteligencia emocional como la combinación de atributos estrechamente relacionados con la personalidad y las competencias ligadas al logro académico y profesional. En general, permite evaluar las características de la experiencia emocional y de la conducta asociada mediante pruebas de autoinforme (Chapman y Hayslip, 2005). Los instrumentos más notables son: el Emotional Quotient inventory (EQ-i) de BarOn (1997) y Emotional Competence Inventory (ECI) de Goleman (1998) y más actualizada el The Emotional and Sociality Inventory (ESCI) Boyatzis, (2007). El primero, lo forman 133 ítems en 15 subescalas agrupadas en cinco dimensiones de orden superior: inteligencia intrapersonal; inteligencia interpersonal; adaptación; manejo del estrés; y de humor general. La consistencia interna de las subescalas oscila entre 0,69 y 0,86 . En la actualidad se está desarrollando una versión reducida del inventario EQ-i: Short con 51 ítems que evalúa las mismas dimensiones. El segundo, nace en la empresa para detectar a los trabajadores con más éxito. Inicialmente se formularon 5 dimensiones de la inteligencia emocional subdivididas en 25 competencias, que posteriormente se redujeron 4 dimensiones y 20 competencias (Boyatzis, Goleman y Rhee, 2000): autoconciencia; automanejo; conciencia social y manejo de las relaciones. Las fiabilidad de la escala varían entre 0,61 y 0,86 en la versión autoinformada y oscila entre 0,79 y 0,94 para la versión de evaluador externo (Boyatzis y Bruckle, 1999). El tercero, es la última herramienta de retroalimentación $360^{\circ}$ sobre la base de otros instrumentos como los mencionados. Describe 12 competencias organizadas en cuatro grupos: auto-conciencia, la autogestión, conciencia social, y gestión de las relaciones (Boyatzis, 2007).

Con el marco teórico de ambos constructos nuestra propuesta de trabajo de investigación, justificándose por el deseo de aportar un instrumento que permita diagnosticar la percepción de las competencias emocionales.

\section{Método}

\section{Objetivos}

El objetivo general del estudio es analizar la percepción de los estudiantes universitarios de educación sobre las competencias emocionales y su relación con algunos factores que expliquen un modelo de competencias aplicable, fiable y válido para su aplicación. Que se concreta en los objetivos específicos:

1. Estimar el grado de fiabilidad de la muestra para medir de forma consistente y precisa las características que se pretenden medir.

2. Analizar las dimensiones que definen las competencias emocionales, atendiendo a los diferentes ítems que forman parte de la muestra de estudio para establecer un modelo explicativo.

3. Evaluar la fiabilidad de las respuestas obtenidas en los sujetos de la muestra. 
4. Analizar la validez del constructo propuesto a través de la validez discriminante y convergente.

5. Contrastar la capacidad de explicación factorial con relación a la media de las distintas titulaciones de la Facultad de Ciencias de la Educación.

\section{Participantes}

La muestra total está compuesta por 571 estudiantes de primer curso de las titulaciones de la Facultad de Ciencias de la Educación de la Universidad de La Coruña (Spain), con edades comprendidas e 17 y 39 años $(M=20,44$ y $\mathrm{SD}=5,012)$.

\section{Instrumento y procedimiento}

Para la elaboración del instrumento se ha tenido en cuenta: 1) Establecer la teoría subyacente al instrumento que permitiese el diseño y construcción del cuestionario, se revisaron las teorías propuestas, especialmente: Bar-On, R. (1997); Bisquerra (200; 2003; Goleman (1996; 1999); Chabot, (2001); Mayer, J. D., y Salovey, P. (1997); entre otros. 2) Se elaboraron las dimensiones y los indicadores concretos que reflejaban la teoría. 3) Se seleccionaron y redactaron las preguntas y el diseño de la estructura del cuestionario. 4) Se sometió al juicio de expertos (profesores universitarios, de enseñanza media y estudiantes universitarios). 4) Una vez realizadas las modificaciones, se aplicó de forma piloto a 44 estudiantes universitarios. Finalmente, el instrumento queda con 72 ítems y 4 de identificación, divididos en 6 bloques o dimensiones, con dos preguntas control y 27 ítems negativos. El Cuestionario de la percepción de las competencias emocionales, nivel superior CUPECE-SUP., es de tipo Lickert con 4 categorías o alternativas de respuesta que van desde nada o nunca hasta mucho o siempre.

El cuestionario se aplicó a los sujetos en horario académico por la autora del estudio, garantizando la identidad de las instrucciones a todos los grupos, y garantizando el anonimato y que las respuestas no tendrían consecuencias en las calificaciones.

\section{Análisis realizados}

Se ha combinando la estadística descriptiva e inferencial: estadísticos de fiabilidad ( $\alpha$ de Cronbach), análisis factorial, matrices de correlaciones y ANOVA multifactorial. El tratamiento y análisis de datos ha sido efectuado mediante el programa estadístico SPSS, versión 15.0 para Windows.

\section{Resultados}

\section{Objetivo primero}

Estimar el grado de fiabilidad de la muestra para medir de forma consistente y precisa las características que se pretenden medir. 
Este objetivo pretende establecer la consistencia interna o grado de coincidencia, y la estabilidad en el tiempo o capacidad del instrumento que sea generalizable. Los datos reflejan valores elevados del coeficiente alfa de Cronbach $(\alpha=.736)$ y del valor tipificado $(\boldsymbol{\alpha}=.765)$.

TABLA 1: Estadísticos de fiabilidad.

\begin{tabular}{|l|c|}
\hline Alfa de Cronbach & .736 \\
\hline Alfa de Cronbach basada en los elementos tipificados & .765 \\
\hline Número de elementos & 72 \\
\hline
\end{tabular}

\section{Objetivo segundo}

Analizar las dimensiones que definen las competencias emocionales, atendiendo a los diferentes items que forman parte de la muestra de estudio para establecer un modelo explicativo.

Se aplicó un análisis factorial, para agrupar las variables en diferentes factores explicativos que formarán índices en base al porcentaje de explicación común de los ítems de estudio. Para ello realizamos los pasos siguientes: 1) Reducción información de 72 variables a 31 , tras elegir las variables con comunalidades con valores $\geq .5$.

Tabla 2: KMO y prueba de Barlett.

\begin{tabular}{|l|c|}
\hline Medida de adecuación muestral de Kaiser-Meyer-Olkin &, 758 \\
\hline Chi-cuadrado aproximado & 3503,895 \\
\hline GI & 465 \\
\hline Sig. &, 000 \\
\hline
\end{tabular}

\section{a) Pruebas de adecuación muestral}

Como se puede apreciar en el estadístico anterior, se muestran dos medidas de adecuación muestral que permiten valorar la magnitud y la precisión del grupo de variables para ser finalmente condensadas en factores.

Con relación a la primera medida de adecuación de la muestra $K M O=.758$, para el conjunto de todas las variables seleccionadas, interpretándose como mediano próximo a meritorio.

Estos datos quedan refrendado, por la segunda medida de adecuación muestral, la prueba de esfericidad de Bartlett, que también mide las correlaciones entre variables, con un elevado valor de $\chi^{2}$ y una $\mathrm{p}>05\left(\chi^{2}=3503,895\right.$ y $\left.\mathrm{p}=.000\right)$.

La tercera medida de adecuación muestral se encuentra en el estadístico denominado Matrices anti-imagen ofreciendo el valor $K M O$ individual $\geq .05$, que refleja la magnitud individual que aporta cada variable al análisis, como por ejemplo destacar los siguientes 
ítems con los valores más elevados: "capto los sentimientos y los puntos de vista de otras personas" (.847), "me gusta innovar y crear cosas nuevas" (.844) y "me esfuerzo para conseguir perfeccionarme en cualquier cosa que emprenda" (.842).

Finalmente, la cuarta medida de adecuación muestral, correlaciones anti-imagen, formada por las KMO individuales, ofreciendo valores bajos y próximos a 0 , implicando así la existencia de no homicidad entre las variables de estudio que podrían ser agrupadas en factores comunes por su información compartida.

\section{b) Extracción y explicación factorial}

El análisis factorial también nos permite establecer un modelo factorial de 10 componentes para explicar la interrelación subyacente a las competencias emocionales de los estudiantes de educación. De las 72 variables iniciales que proporcionaba el cuestionario hemos llegado a 31 componentes que explicarían el 100\% de la interrelación de todas las variables, pero, finalmente, nos quedamos con 10 factores que explican un $60 \%$ la interrelación subyacente de las variables de estudio. (Se omite la tabla por extensa).

Los criterios de selección de los factores han sido: 1) Criterio de autovalores mayores que uno: 10 factores con un autovalor máximo $=1,064>1$. 2) Criterio de varianza explicada: porcentaje acumulado con 10 factores $(10=59,364)$. 3) Criterio de contraste de caída evaluado en el gráfico de sedimentación (se omite por la extensión del artículo).

La explicación factorial está avalada por el estadístico matriz de componentes rotada, mediante el método de rotación Varimax. Sólo se han seleccionado las variables con puntuaciones factoriales $\geq$ a .05. (Se omite la tabla por extensa):

- $1^{\circ}$ factor denominado Innovación y creatividad, consta de de tres ítems: 1. "me gusta cambiar y crear cosas nuevas" (.753); 2. "todas las cosas nuevas me fascinan porque me gusta experimentar" (.730); 3. "me gusta innovar y ver las cosas desde otro punto de vista" (.673). Todas las variables superan notablemente el mínimo requerido .05 destacando el primer ítem que da nombre el factor. El coeficiente de fiabilidad, consistencia interna alfa de Cronbach, para los elementos que integran el factor es $\alpha=.701$, avalando el contenido del factor. En los demás factores sólo se pondrá el valor de alfa de Cronbach.

- $2^{\circ}$ factor denominado Habilidades sociales, consta de tres ítems: 1. "se escuchar a mis amigos, pareja o familia y comprenderles" (.738); 2. "capto los sentimientos y los puntos de vista de las otras personas y me intereso por ello" (.702); 3. "me doy cuenta de las necesidades y preocupaciones de mis familiares" (.683). Con un valor $\alpha=.643$.

- $3^{\circ}$ factor denominado Autocontrol, consta de 4 ítems: 1. "tengo que hacer muchos esfuerzos para no perder la calma" (.706); 2. "me enfado mucho cuando me cambian los planes que tenía hechos" (.677); 3. "me cuesta controlar el enfado o el miedo" (.543); 4. "si las cosas van mal me desmotivo" (.501). Con un valor $\alpha=.562$.

- $4^{\circ}$ factor denominado Autoestima, consta de 2 ítems: 1. "me gusta mi cuerpo tal como es" $(0,750) ; 2$. "estoy satisfecho conmigo como persona" (.732). Con un valor $\alpha=.582$, este valor se justifica porque son solo dos variables. 
- $5^{\circ}$ factor denominado Comunicación, consta 2 ítems: 1. "puedo exteriorizar mi mundo interior con mis familiares y amigos" (.848); 2. "comento con mis amigos, pareja o familia" (.807). Con un valor $\alpha=.731$.

- $6^{\circ}$ factor denominado Responsabilidad, consta de 2 ítems: 1. "siempre cumplo los compromisos" (.861); 2. "siempre cumplo mis compromisos con mi familia..." (.778). Con un valor $\alpha=.718$.

- $7^{\circ}$ factor denominado Resolución de conflictos, consta de 3 ítems: 1. "cuando hay un conflicto lo mejor es buscar soluciones" (.708); 2. "respeto la diversidad personal y sus necesidades" (.589); 3. "cuando surge un conflicto lo mejor es tratar de llegar a un acuerdo" (.569). Con un valor $\alpha=.420$, este valor podría explicarse, quizá, por las dos variables finales son menos significativas.

- $8^{\circ}$ factor denominado Adaptación laboral, consta de 2 ítems: 1. "prefiero que me den una orden y cumplirla" (.759); 2. "en el trabajo o estudios prefiero que me digan lo que tengo que hacer sin complicarme mas" (.670). Con valor $\alpha=.508$ con un valor aceptable por ser dos variables.

- $9^{\circ}$ factor denominado Tolerancia a la frustración, consta de 1 sólo ítem: 1. "si no consigo lo que pretendía no pienso mas en ello" (.865). Esta variable tiene el suficiente peso para constituir un factor con su nombre. Esta variable se ha utilizado como control en el cuestionario para contrastar la fiabilidad de respuesta de los alumnos y el valor obtenido de fiabilidad la refuta (.841). Con un valor $\alpha=.495$, valor justificado por ser únicamente una variable y llegar casi al .05.

- $10^{\circ}$ factor denominado Autoconciencia, es el último y consta de 2 ítems: 1. "soy consciente de mis pensamientos" (.737); 2. "reconozco las emociones y sentimientos que..." (.668). Con un valor $\alpha=0,448$, valor se justifica por ser solo dos variables y la segunda con menor carga factorial.

Destacamos los factores con el coeficiente de fiabilidad más elevados por orden de estudio: $1^{\circ}$ factor Innovación y creatividad $\alpha=0,701 ; 2^{\circ}$ factor Comunicación $\alpha=0,731$ y $3^{\circ}$ factor Responsabilidad $\alpha=0,718$.

\section{Objetivo tercero}

\section{Evaluar la fiabilidad de las respuestas obtenidas en los sujetos de la muestra.}

Para evaluar la fiabilidad de la respuesta de los sujetos que forman la muestra de estudio, se aplicó el criterio de control de variables-respuesta, con dos ítems repetidos, a lo largo del cuestionario referidos al compromiso y a la tolerancia a la frustración:

Ítems compromiso: "siempre cumplo los compromisos" y "siempre cumplo los compromisos que adquiero con mi familia, amigos, pareja y familia". Los datos obtenidos de este análisis de correlación de Pearson constatan una correlación en ambos ítems-respuesta, muy similar y óptimo con un valor de $\mathrm{R}=(.560)$, y una probabilidad asociada.

Ítems tolerancia a la frustración: se repitió la misma variable con matices de redacción para verificar si la respuesta obtenida fue la misma o parecida "si no consigo lo que pretendía no pienso más en ello" y "si no consigo lo que pretendía. No pienso más en ello". Los datos obtenidos de este análisis de correlación de Pearson constatan una correlación en am- 
bos ítems-respuesta, muy similar con un valor de $\mathrm{R}=(.466)$, y una probabilidad asociada $.000 \leq .01$ a nivel bilateral.

TABLA 3. Matrices de correlación: control de variable respuesta primer ítem.

\begin{tabular}{|l|c|c|}
\cline { 2 - 3 } \multicolumn{1}{c|}{} & $\begin{array}{c}\text { Siempre cumplo } \\
\text { los compromisos }\end{array}$ & $\begin{array}{c}\text { Siempre cumplo mis } \\
\text { compromisos que adquiero } \\
\text { con mi familia... }\end{array}$ \\
\hline Correlación de Pearson & 1 & $.560\left(^{* *}\right)$ \\
\hline Sig. (bilateral) & 614 & .000 \\
\hline N & $.56\left(^{* *}\right)$ & 614 \\
\hline Correlación de Pearson & .000 & 1 \\
\hline Sig. (bilateral) & 614 & 720 \\
\hline N & \multicolumn{2}{|c|}{} \\
\hline
\end{tabular}

TABLA 4. Matrices de correlación: control de variable respuesta segundo ítem.

\begin{tabular}{|l|c|c|}
\cline { 2 - 3 } \multicolumn{1}{c|}{} & $\begin{array}{c}\text { Si no consigo lo que } \\
\text { pretendía no pienso } \\
\text { más en ello. Repe }\end{array}$ & $\begin{array}{c}\text { Si no consigo lo que } \\
\text { pretendía. No pienso } \\
\text { más en ello }\end{array}$ \\
\hline Correlación de Pearson & 1 & $\left..466^{* *}\right)$ \\
\hline Sig. (bilateral) & 615 & .000 \\
\hline N & $.466\left(^{* *}\right)$ & 609 \\
\hline Correlación de Pearson & .000 & 1 \\
\hline Sig. (bilateral) & 609 & 714 \\
\hline N & 609 & \\
\hline
\end{tabular}

** La correlación es significativa al nivel 0,01 (bilateral).

\section{Objetivo cuarto}

Analizar la validez del constructo propuesto a través de la validez discriminante y convergente.

\section{Validez discriminante}

Para evaluar la validez discriminante del instrumento se procedió a clasificar a los sujetos en dos grupos en función de la media obtenida en base al sumatorio total de las puntuaciones obtenidas en cada ítem. Así surgen dos grupos: $1^{\circ}$ el grupo que está por debajo de la media; y el $2^{\circ}$ grupo que está por encima de la media. 
El estadístico Anova de un factor nos proporciona el contraste de medias grupales para la variable totimes (total de ítems) que refleja la puntuación media de los casos en los factores previamente elegidos. El nivel de significación encontrado para todos los factores es estadísticamente óptimo $<.05(\mathrm{p}=.000<.05)$, quedando validada la hipótesis de que las diferencias medias grupales son diferentes, lo que significa que las variables discriminan de distinta manera a los dos grupos establecidos previamente, confirmando su validez discriminante.

\section{Validez convergente}

Para evaluar la validez convergente del instrumento, se procedió a elaborar una matriz de correlaciones que permitiera evaluar el grado de asociación entre el total de puntuaciones obtenidas por los estudiantes, los factores y las "preguntas control" incluidas en el cuestionario. El coeficiente de correlación lineal $\mathrm{r}$ de Pearson presenta un óptimo nivel de significación $(\mathrm{p}=.000)$ para todos los factores y las preguntas control.

TABLA 5. Correlación de los factores.

\begin{tabular}{|l|c|c|c|c|c|c|c|c|c|c|c|c|}
\hline Total ítems caso & 1 & 2 & 3 & 4 & 5 & 6 & 7 & 8 & 9 & 10 & 1 & 2 \\
\hline Correlación de Pearson & 0.336 & 0.162 & 0.460 & 0.314 & 0.135 & 0.407 & 0.170 & 0.323 & 0.304 & 0.186 & 0.336 & 0.162 \\
\hline Sig. (bilateral) & 0.000 & 0.000 & 0.000 & 0.000 & 0.000 & 0.000 & 0.000 & 0.000 & 0.000 & 0.000 & 0.000 & 0.000 \\
\hline
\end{tabular}

\section{Objetivo quinto}

Contrastar la capacidad de explicación factorial con relación a la media de las distintas titulaciones de la Facultad de Ciencias de la Educación.

Para evaluar la capacidad de explicación factorial del instrumento, con relación a la media de las distintas titulaciones analizadas y los factores obtenidos, se procedió a realizar un Análisis de varianza (ANOVA multifactorial). Se constata que todos los factores son buenos discriminantes ya que presentan un valor de $F>3,84$ y una $p=.000$. A modo de ejemplo, destacamos aquellos más significativos: habilidades sociales $(\mathrm{F}=185,849$; $\mathrm{p}=.000) ;$ innovación y creatividad $(\mathrm{F}=163,078 ; \mathrm{p}=.000) ;$ autoestima $(\mathrm{F}=131,066$; $\mathrm{p}=.000) ;$ autocontrol $(\mathrm{F}=127,695 ; \mathrm{p}=.000)$ y responsabilidad $(\mathrm{F}=64,559 ; \mathrm{p}=.000)$.

\section{Conclusiones}

De lo expuesto anteriormente podemos concluir que el CUPECE-SUP cumple satisfactoriamente con la función de diagnóstico de las competencias emocionales propuesto en el objetivo general de nuestra investigación. El instrumento elaborado podría encuadrarse en la categoría de los modelos de rasgo o mixtos de Bar-On (1997) y Goleman (1999), estudiados en la introducción según las categorías de Mayer, Salovey y Caruso (2000). La consistencia interna del EQ-i oscila entre .69 y .86, mientras que el ECI lo hace entre .61 y .86. Nuestro instrumento está dentro de esa banda, con una consistencia interna óptima $(\boldsymbol{\alpha}=.765)$ para todo el cuestionario. 
TABLA 6. Anova.

\begin{tabular}{|c|c|c|c|c|c|c|}
\hline & & $\begin{array}{l}\text { Suma de } \\
\text { cuadrados }\end{array}$ & gl & $\begin{array}{c}\text { Media } \\
\text { cuadrática }\end{array}$ & $\mathbf{F}$ & Sig. \\
\hline Innovación y creatividad & $\begin{array}{l}\text { Inter-grupos } \\
\text { Intra-grupos } \\
\text { Total }\end{array}$ & $\begin{array}{r}137,936 \\
1396,462 \\
1534,399\end{array}$ & $\begin{array}{r}1 \\
1651 \\
1652\end{array}$ & $\begin{array}{r}137,936 \\
, 846\end{array}$ & 163,078 &, 000 \\
\hline Habilidades sociales & $\begin{array}{l}\text { Inter-grupos } \\
\text { Intra-grupos } \\
\text { Total }\end{array}$ & $\begin{array}{r}167,414 \\
1487,232 \\
1654,646\end{array}$ & $\begin{array}{r}1 \\
1651 \\
1652 \\
\end{array}$ & $\begin{array}{r}167,414 \\
, 901\end{array}$ & 185,849 &, 000 \\
\hline Autocontrol & $\begin{array}{l}\text { Inter-grupos } \\
\text { Intra-grupos } \\
\text { Total }\end{array}$ & $\begin{array}{r}121,474 \\
1570,566 \\
1692,041 \\
\end{array}$ & $\begin{array}{r}1 \\
1651 \\
1652 \\
\end{array}$ & $\begin{array}{r}121,474 \\
, 951\end{array}$ & 127,695 & ,000 \\
\hline Autoestima & $\begin{array}{l}\text { Inter-grupos } \\
\text { Intra-grupos } \\
\text { Total }\end{array}$ & $\begin{array}{r}120,235 \\
1514,571 \\
1634,807\end{array}$ & $\begin{array}{r}1 \\
1651 \\
1652\end{array}$ & $\begin{array}{r}120,235 \\
, 917\end{array}$ & 131,066 & ,000 \\
\hline Comunicación & $\begin{array}{l}\text { Inter-grupos } \\
\text { Intra-grupos } \\
\text { Total }\end{array}$ & $\begin{array}{r}13,745 \\
1658,198 \\
1671,943\end{array}$ & $\begin{array}{r}1 \\
1651 \\
1652\end{array}$ & $\begin{array}{r}13,745 \\
1,004\end{array}$ & 13,685 & 000 \\
\hline Responsabilidad & $\begin{array}{l}\text { Inter-grupos } \\
\text { Intra-grupos } \\
\text { Total }\end{array}$ & $\begin{array}{r}63,285 \\
1617,403 \\
1680,688\end{array}$ & $\begin{array}{r}1 \\
1651 \\
1652\end{array}$ & $\begin{array}{r}63,285 \\
, 980\end{array}$ & 64,599 & 000 \\
\hline Resolución conflictos & $\begin{array}{l}\text { Inter-grupos } \\
\text { Intra-grupos } \\
\text { Total }\end{array}$ & $\begin{array}{r}27,383 \\
1522,114 \\
1549,497\end{array}$ & $\begin{array}{r}1 \\
1651 \\
1652 \\
\end{array}$ & $\begin{array}{r}27,383 \\
, 922\end{array}$ & 29,701 &, 000 \\
\hline Adaptación & $\begin{array}{l}\text { Inter-grupos } \\
\text { Intra-grupos } \\
\text { Total }\end{array}$ & $\begin{array}{r}35,488 \\
1591,020 \\
1626,508\end{array}$ & $\begin{array}{r}1 \\
1651 \\
1652 \\
\end{array}$ & $\begin{array}{r}35,488 \\
, 964\end{array}$ & 36,826 & ,000 \\
\hline Tolerancia a la frustración & $\begin{array}{l}\text { Inter-grupos } \\
\text { Intra-grupos } \\
\text { Total }\end{array}$ & $\begin{array}{r}41,994 \\
1597,556 \\
1639,551\end{array}$ & $\begin{array}{r}1 \\
1651 \\
1652\end{array}$ & $\begin{array}{r}41,994 \\
, 968\end{array}$ & 43,399 & ,000 \\
\hline Autoconciencia & $\begin{array}{l}\text { Inter-grupos } \\
\text { Intra-grupos } \\
\text { Total }\end{array}$ & $\begin{array}{r}35,313 \\
1506,043 \\
1541,356\end{array}$ & $\begin{array}{r}1 \\
1651 \\
1652\end{array}$ & $\begin{array}{r}35,313 \\
, 912\end{array}$ & 38,712 & ,000 \\
\hline
\end{tabular}

De los datos obtenidos parecen confirmar la fiabilidad del cuestionario ya que mide de forma consistente, precisa y sin error la característica que pretende medir (Pardo y Ruiz, 2005: 507). En este sentido se ha cumplido el objetivo de fiabilidad del cuestionario, también, la fiabilidad de la respuesta que los sujetos de la muestra han proporcionado al utilizar dos pares de ítems repetidos: a) con respecto a las variables de compromiso y, b) con el par de ítems de tolerancia a la frustración.

Mientras que la validez es la capacidad de la escala para medir lo que pretende medir. Dentro de este concepto se encuadra la validez discriminativa o capacidad de diferenciar entre sujetos que difieren en la característica medida (Pardo y Ruiz, 2005: 507). La validez discriminativa del cuestionario alcanza un nivel de significación óptima para todos los factores, implicando las medias grupales de la muestra de estudiantes de educación analizada son diferentes. La validez convergente se establece a partir de correlaciones altas y significativas entre las variables manifiestas y las variables latentes (Corral, 1995; Hair, Anderson, Tatham y Black, 1999); en nuestra investigación hemos obtenido un valor del coeficiente de 
correlación lineal $\mathrm{r}$ de Pearson óptimo para todos los factores y las preguntas de control. La validez estructural o factorial pretende averiguar el grado en que los indicadores de un concepto reflejan o son parte de dicho concepto. Para cada faceta o dimensión hay varios ítems o indicadores, la validez estructural o factorial intenta discernir el grado en que los indicadores de un concepto reflejan dicho concepto, son parte de él o lo constituyen.

Por otra parte, los datos obtenidos confirman la bondad de la adecuación muestral, implicando, así, la existencia de suficientes correlaciones entre las variables de estudio para interrelacionarse en diferentes componentes. Por tanto, es factible un modelo explicativo de 10 componentes para diagnosticar la percepción de las competencias emocionales en los estudiantes universitarios de educación: Innovación y creatividad, Habilidades sociales, Autocontrol, Autoestima, Comunicación, Responsabilidad, Resolución de conflictos, Adaptación, Tolerancia a la frustración y Autoconciencia. Estos factores se encuentran en el Proyecto Tunning (González y Wagenaar, 2003) en las competencias genéricas (interpersonales y sistémicas), así como en las dimensiones de la inteligencia emocional en diferentes autores, en algunos sólo aparecen parte de los factores mencionados y en otros, la totalidad, pero la idea común es detectar aquellos aspectos que más desarrollan al ser humano en todas sus facetas (Bar-On (1997); Boyatzis and Bruckle (1999); Chabot (2001); Goleman (1996; 1998); Mayer, and Salovey (1997).

Finalmente, la validez de la capacidad de explicación factorial con relación a las medias de las distintas titulaciones de Ciencias de la Educación confirman la bondad de explicación de los 10 factores propuestos Siendo los factores más discriminantes: habilidades sociales, innovación y creatividad, autoestima y autocontrol.

Para investigaciones futuras consideramos que podrían abordarse varios aspectos relacionados con: a) la elaboración programas de intervención para mejorar las capacidades emocionales de los docentes y así generar nuevas competencias desde la escuela en beneficio de toda la sociedad; b) ampliar las competencias estudiadas desde el marco del Proyecto Tunning (González y Wagenaar, 2003), considerando, también, las competencias profesionales; y c) relacionar estas competencias con las emociones básicas que modifican la conducta cotidianamente.

Finalmente, ejerciendo la autocrítica, debemos considerar las limitaciones del cuestionario, que puede producir sesgos en función de lo socialmente deseable o lo que se desea decir sobre las propias percepciones de las competencias emocionales.

\section{Referencias bibliográficas}

Bar-On, R. (1997). EQ-i Bar-On Emotional Quotient Inventory: A measure of emotional inventory. Technical manual. Canada: Multi-Health Systems.

Bisquerra, R. (2000). Educación emocional y bienestar. Barcelona: Praxis.

Bisquerra, R. (2003). Educación emocional y competencias básicas para la vida. Revista de Investigación Educativa (RIE), 21, 1, 7-43.

Boyatzis, R. (2007). "The Creation of the Emotional and Social Competency Inventory (ESCI)”. Hay Group: Boston.

Boyatzis, R. E. and Bruckle, M. (1999). Psychometric propierties of the ECI: Technical Note. Boston: The Hay/McBer Group. 
Boyatzis, R.; Goleman, D. and Rhee, K. (2000). Custering competence in emotional intelligence. Insights from the emocional competence inventory (ECI). En Bar-On y J. D. A. Parker (Eds.), Handbook of emotional intelligence (343-367). San Francisco: Jossey-Bass.

Corral, V. (1995). Modelos de variables latentes para la investigación conductual. Acta Comportamentalia, 3, 171-190.

Chabot, D. (2001). Cultive su inteligencia emocional. Bilbao: Edición Mensajero.

Chapman, B. and Hayslip, J. (2005). Incremental validity of a measure of emotional intelligence. Journal of Personality Assessment, 85(2), 154-169.

Declaración de Bolonia (1999). El Espacio Europeo de la Enseñanza Superior. Declaración conjunta de los ministros europeos de educación reunidos en Bolonia el 19 de junio de 1999. http://www. eees.es/pdf/Bolonia_ES.pdf.

Goleman, D. (1996). La inteligencia emocional. Buenos Aires: Javier Vergara Editor.

Goleman, D. (1998). Working with emotional intelligence. New York: Bantan Books. (Trad. Cast. Kairós, 1999).

Goleman, D. (1999). La inteligencia emocional en la empresa. Buenos Aires: Javier Vergara Editor.

González, J. and Wagenaar, R. (2003). Tuning Educational Structures in Europe. Final Report. Phase One. Madrid: Universidad de Deusto. Versión electrónica: http://www.scribd.com/doc/17811793/ Proyecto-Tuning-Europa.

Hair, J., Anderson, R., Tatham, R., \& Black, W. (1999). Análisis Multivariante (5 edición). Madrid: Prentice Hall Iberia.

Le Boterf, G. (2001). Ingeniería de las competencias. Barcelona: Gestión 2000.

Levy-Leboyer, C. (1997). Gestión de las competencias: cómo analizarlas, cómo evaluarlas, cómo desarrollarlas. Barcelona: Gestión 2000.

Lopes, P. N.; Brackett, M. A.; Nezlek, J. B.; Schütz, A.; Sellin, I. and Salovey, P. (2004). Emotional intelligence and social interaction. Personality and Social Psychology Bulletin, 30(8), 1018-1034.

Mayer, J. D. and Salovey, P.(1997). What is emotional intelligence? En P. Salovey and D. Sluyter (Eds.), Emotional development and emotional intelligence: Educational implications (pp. 3-31). New York: Basic Books.

Mayer, J. D.; Salovey, P. and Caruso, D.R. (1999). Mayer, Salovey, Caruso Emotional Intelligence Test: Research version 1.1 Manual (2da ed.). Canada: Multi-Health Systems.

Mayer, J. D.; Salovey, P. and Caruso, D.R. (2000). Models of emotional intelligence. En R.J. Sternberg (Ed), The handbook of intelligence (2da ed., pp. 396-420). New York: Cambridge University Press.

Pardo, A. y Ruiz, M. A. (2005). Análisis de datos con SPSS 13 base. Madrid: McGraw Hill.

Salovey, P. and Mayer, J. (1990). Emotional intelligence. Imation, Cognition and Personality, 9(3), 185-211.

Fecha de recepción: 15-07-2009

Fecha de revisión: 29-09-2009

Fecha de aceptación: 21-10-2009 